\title{
Arthrodesis After Infected Revision TKA: Retrospective Comparison of Intramedullary Nailing and External Fixation
}

\author{
Iacono Francesco, MD • Raspugli Giovanni Francesco, MD • Bruni Danilo, MD • \\ Lo Presti Mirco, MD • Sharma Bharat, MD • \\ Akkawi Ibrahim, MD • Marcacci Maurilio, MD
}

Received: 18 March 2013/Accepted: 3 July 2013 / Published online: 14 August 2013

(C) Hospital for Special Surgery 2013

\begin{abstract}
Background: Infection after revision total knee arthroplasty (TKA) for previous septic TKA can be a challenging problem to treat due to loss of bone stock and soft tissue integrity. In these cases, arthrodesis is a well-recognized salvage procedure. Questions/Purposes: The aim of this retrospective study was to compare the results as described by a Visual Analogue Scale (VAS) and the Lequesne Algofunctional Score (LAS) of knee arthrodeses performed by using either an external fixator (EF) or an intramedullary nail (IM). Methods: The study included 34 knee arthrodesis divided in two groups: first group included 12 patients treated with $\mathrm{EF}$ and the second group of 22 patients dealt with IM nail. Clinical and functional evaluation was performed using the VAS and the LAS. Full-length radiographs were used to verify limb length discrepancy. Results: VAS and LAS results showed a substantial improvement relative to preoperative condition in both groups. However, the LAS was significantly better in the IM nail group. The mean leg length discrepancy was significantly greater $(4.5 \mathrm{~cm})$ in the first group than in the second one $(0.8 \mathrm{~cm})$. No recurrence of infection was observed in the EF group while there were three recurrent infections in the IM nail group. Conclusion: Our study supported the existing literature and found that reinfection after revision TKA can be
\end{abstract}

$\overline{\text { Level of Evidence: Therapeutic Study Level III. See levels of evidence }}$ for a complete description.

Electronic supplementary material The online version of this article (doi:10.1007/s11420-013-9349-5) contains supplementary material, which is available to authorized users.

I. Francesco, MD · R. G. Francesco, MD · B. Danilo, MD $(\bowtie) \cdot$

L. P. Mirco, MD $\cdot$ S. Bharat, MD $\cdot$ A. Ibrahim, MD •

M. Maurilio, MD

Biomechanics Laboratory,

Rizzoli Orthopaedic Institute,

Via di Barbiano n.1/10, Bologna, 40126, Italy

e-mail: d.bruni@biomec.ior.it effectively treated with arthrodesis. In presence of massive bone loss, we recommend arthrodesis with IM nail used as an endoprosthesis, without bone-on-bone fusion, to produce a stable and painless knee, while preserving the limb length. Use of an IM nail allowed us to get a better functional result than EF.

Keywords revision surgery. total knee arthroplasty. infection ·arthrodesis $\cdot$ IM nail $\cdot E F \cdot$ limb salvage

\section{Introduction}

Management of an infected total knee arthroplasty (TKA) is a challenging problem faced by orthopedic surgeons and the optimum treatment of the infected TKA is still unclear.

Revision for infection after previous revision TKA (RTKA) for septic TKA adds further challenges to treatment of these difficult cases due to loss of bone stock, soft tissue integrity, and comorbid conditions $[1,17]$. Additionally, the rate of failure following re-revision of an infected R-TKA is considerable [8, 10, 17, 19, 20, 23], while functional results may be lower than those of a successful arthrodesis of the knee $[9,25,28,30]$. Therefore, in these challenging cases, knee arthrodesis may be considered a valid therapeutic option.

The two most frequent arthrodesis techniques are intramedullary (IM) nailing and external fixation (EF). There are advantages and disadvantages for each of these two techniques. Advantages of EF include the ability to produce excellent compression at the femoro-tibial interface and to modify the alignment postoperatively. More importantly, no implants remain in the knee following fixator removal $[12,16]$. Disadvantages of EF include pin site infection, fracture at a pin site, the need to avoid immediate weight bearing, prolonged application to achieve fusion, and lower rate of fusion $[6,12,16,26,27,29]$. Benefits of intramedullary nail include great stability $[11,21]$, faster full 
weight bearing $[3,24]$, higher rate of fusion $[4,8,16,21$, $24]$, and better tolerance by the patient than EF [6, 8]. Both these techniques fuse the joint through direct bone to bone apposition, often resulting in leg length discrepancy which may be a cause of functional impairment and discomfort during ambulation.

In our department, external fixation was historically the preferred technique for knee arthrodesis following infected TKR. Typically, a complete bone-to-bone fusion was achieved without the need of multiple reoperations, but a mean limb shortening of $4.5 \mathrm{~cm}$ was typical. To address the problem of the limb length discrepancy, we shifted to the intramedullary nailing technique, using a press-fit modular nail as an endoprosthesis without bone-on-bone contact. Moreover, the residual gap between the tibia and the femur was filled with an antibiotic-loaded cement spacer to potentially minimize the risk of reinfection.

The object of this retrospective study was to compare these two approaches to arthrodesis following failed, infected TKR. Specifically, we aimed to detect any difference in recurrence of infection and in leg length discrepancy. Additionally, we wished to compare the pain (Visual Analogue Scale), the function results as assessed by the Lequesne Algofunctional Score, and the patient's satisfaction, of a consecutive group of 34 patients, who underwent knee arthrodesis using either of these techniques for an infected R-TKA

\section{Material and Methods}

In this retrospective study, we analyzed all patients who underwent knee arthrodesis for infected R-TKA in our department between 2001 and 2009. We divided the patients into two groups: the first group included 12 patients who were treated with an external fixator device, Hoffmann II Stryker (Selzach, Switzerland), between 2001 and 2004, while the second group included 22 consecutive patients who underwent knee arthrodesis using a long IM nail (Link Laboratory, Hamburg, Germany) between 2004 and 2009.

Our series included 19 females and 15 males. The average age of the patients at the time of arthrodesis was 68.9 years, ranging from 53 to 85 years. Two patients in the first group and one patient in the second group died, for unrelated reasons before final follow-up and were excluded from the series, so 31 patients remained for the study.

The mean follow-up was 93.2 months (82-110 months) in patients treated with external fixator and 34.4 months (13-72 months) in patients treated with IM nail.

The mean number of surgical procedures on the affected knee joint before the arthrodesis was 4.6 (range 1-6) in the group treated with IM nail and 3.87 (range 2-6) in the patients treated with external fixator. A "two-stage procedure" was used for all cases of reinfection after R-TKA.

The first stage consisted of surgical debridement, component removal, and insertion of an antibiotic-coated cement spacer. We used Palacos bone cement (Biomet, Warsaw, Indiana), with $2 \mathrm{~g}$ of vancomycin per pack of cement. Bone samples and fibrous tissue from the femur, tibia, and the joint were taken for microbiological examination. Postoperative intravenous antibiotics were administered according to the culture results, or empirically, for 6 weeks. Antibiotics were discontinued after 6 weeks. If a normalization of the blood parameters indicative of infection occurred (CRP, ESR, and WBC) after 4 weeks, we performed the arthrodesis.

In the external fixation group, the second stage consisted of spacer removal, saw resection of joint surfaces to correct alignment, providing maximum contact area and best external fixator positioning (Fig. 1). The patella was left alone in seven cases. In three cases, it was used to augment the fusion mass and to improve the antero-posterior stability. In all cases, an axial external fixator, the Hoffmann II Stryker (Salzach, Switzerland), was used.

The number of pins used with the Hoffmann II fixator varied. We normally used three transfixion pins in the femur and three in the tibia: one anteriorly, one laterally, and the last one medially.

The transfixion pins were connected using semicircular curved rod. Other two semicircular curved rod were placed by same technique, $5 \mathrm{~cm}$ proximally and $5 \mathrm{~cm}$ distally to previously implanted circular rod. The femoral curved rods were connected with each other, as well the tibial curved rods. The average distance between the most distal femoral semicircular rod and the most proximal tibial semicircular rod was $15 \mathrm{~cm}$.

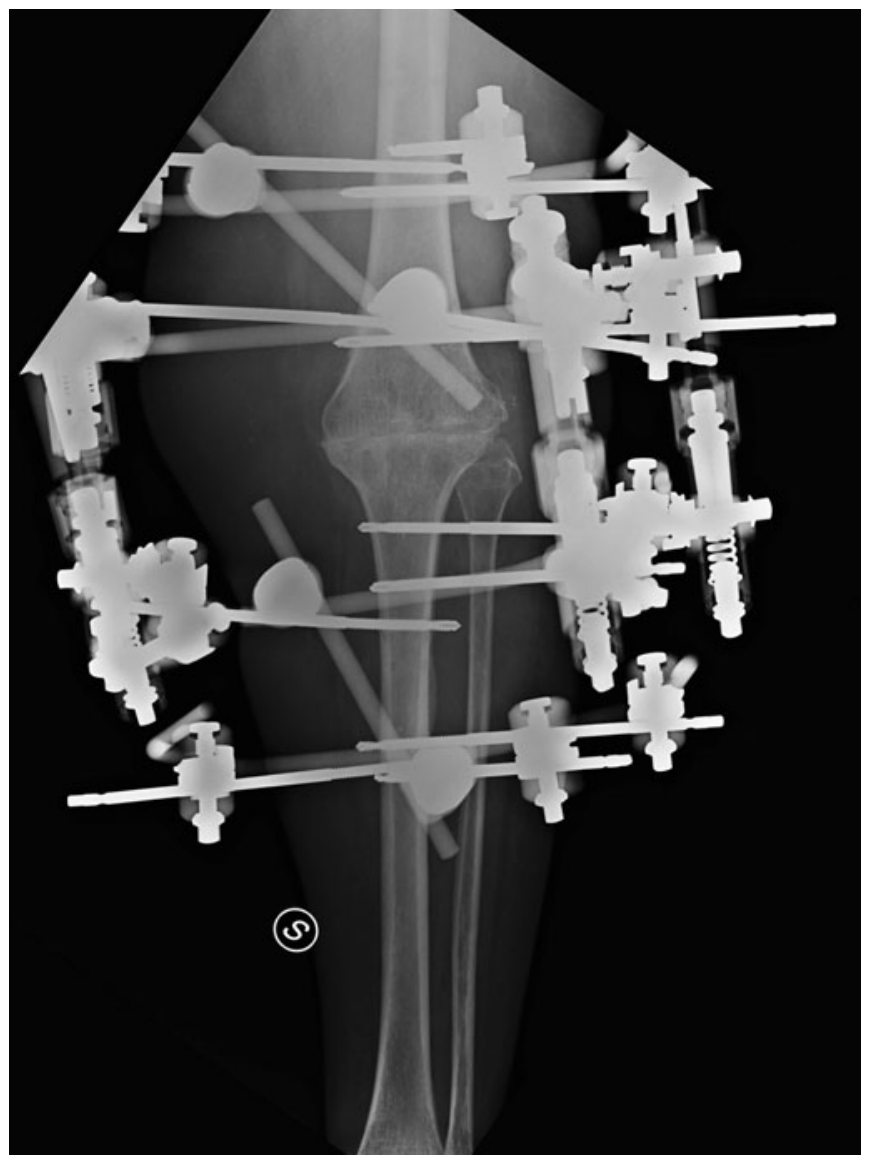

Fig. 1. Frontal radiograph of a 72-year-old woman with an infected revision TKA treated with EF. Good bone contact and proper alignment was achieved. 
Once bony apposition and the alignment were satisfactory, the fixator was secured using four compression/distraction rods. In the following months, the compression was adjusted via the compression rods under radiographic control. The ideal alignment was to obtain fusion with the knee in $5-7^{\circ}$ of valgus and $5-15^{\circ}$ of flexion (Fig. 1).

In the other group, the second stage consisted of spacer removal, further debridement and intramedullary nail. In all patients, we used the Link nail (Link Laboratory, Hamburg, Germany) (Fig. 2a, b).

It is composed of two separate femoral and tibial components made of surface-sanded titanium and it is implanted with a cementless technique. During the reaming procedure, we searched for the best possible anatomic fit to the tibial and femoral medullary canals. Primary stability was ensured by the surface-sanded titanium, which also ensured secondary stability by promoting bone growth in contact with titanium. Finally, the two components were assembled by reciprocal interlocking of their base plates and two anteroposterior screws to lock the assembly. The assembly screws have a hole in the threaded section to place an ultra-high molecular weight polyethylene peg before securing the screws, to prevent the screws from backing out. Once assembled, the nail has $5^{\circ}$ valgus angle in the frontal plane and $5^{\circ}$ flexion in the sagittal plane. The controlateral limb length was used as the target length for the arthrodesed limb. We approximated the bone gap between the femur and tibia with Palacos cement impregnated with $4 \mathrm{~g}$ of vancomycin per pack of polymethylmethacrylate (Fig. 2a, b).

After surgery, full weight bearing was allowed within a week in patients treated with IM nailing. Patients treated with external fixator used crutches for partial weight bearing for 2 months, followed by full weight bearing according to the pain tolerance. Postoperative intravenous antibiotics were administered according to the preoperative culture results, or empirically, for 4 weeks followed by oral antibiotics for 2 weeks.

A retrospective evaluation was performed at final followup by two different surgeon (BD, RG) using the Visual Analogue Scale (VAS) for self-assessment of pain (on a 0 10 scale, with 0 for no pain and 10 as worst pain ever experienced) and the Lequesne Algofunctional Score (LAS) [14] to assess the severity of handicap in their daily life. Low Lequesne Algofunctional score corresponds to good results. Limb length discrepancy along with the use of any compensatory device was noted.

We also enquired about patient satisfaction (rated as very satisfied, fairly satisfied, or unsatisfied) [15] with knee arthrodesis and pain in ipsilateral or contralateral joints such as the hip or the ankle.

In the first group (EF), union was defined clinically as lack of movement at the arthrodesis site when stressed manually after the connective rods were removed from the fixator, with the pins in situ and by painless weight bearing. Union was also defined radiographically as osseous bridging on both antero-posterior and lateral radiographs. In both groups, full-length radiographs were used to verify lower limbs length. Reinfection after arthrodesis was considered as failure of the procedure. We considered reinfected knee when abnormal serology (ESR $>30 \mathrm{~mm} / \mathrm{h}$ and CRP $>1 \mathrm{mg}$ / dl) was observed associated to recurrent chronic pain with tenderness and presence of sinus tracts.

Differences in rate of reinfection were analyzed with the Chi square test. Values of VAS, limb length discrepancy, and LAS were compared between the two groups using Student's $t$ test for independent variables. Post hoc power analysis showed a power of $>0.8$ for dismetry and Lequesne score, while for VAS score it resulted 0.2 .

\section{Results}

Union of the arthrodesis site for the Ex Fix group was achieved in nine cases, and in one patient we had a painless nonunion. The mean time to fusion in the EF group was 5.6 months (range 3.5-11 months). The EF was removed at a mean time of 35 days after fusion (range 23-64 days).

Pin tract infections developed in four patients $(40 \%)$ during treatment, but only two needed surgical intervention, one pin exchange and one fixator removal, before complete bone fusion. In this case, temporary immobilization for 45 days using a cast plaster was required. Infections in the remaining two patients resolved using local pin site care and oral antibiotics.

There was a trend suggesting that the incidence of recurrent infection was higher in the IMN group $(p=0.18)$. Excluding fixator removal, none of patients in the Ex Fix group underwent a reoperation for recurrence of infection. In the IMN group, three recurrent infections after arthrodesis were observed (failure rate 14\%). In all three cases, abnormal and elevated sedimentation rate and CRP with recurrent knee pain and presence of sinus tract were found. In two of these cases, cultures obtained at time of nail removal showed persistence of prior infection, whereas in one case the isolated organism was different from the previous infecting bacteria.

An above-knee amputation was performed in all of three patients, after nail removal, between 4 and 5 years following attempted arthrodesis.

Patients treated with the Ex Fix had greater leg length discrepancy $(p=0.0001)$. The mean leg length discrepancy (shortening) was $4.5 \mathrm{~cm}$ (range 3.5-5.5) in the Ex Fix group compared to $0.8 \mathrm{~cm}$ (range $0-2.2 \mathrm{~cm}$ ) in the IMN group (Table 1).

We did not find any difference in the VAS between the two groups. In the external fixator group, the mean VAS for pain was 1.9 (range 0-7) and four patients (40\%) had no pain $(\mathrm{VAS}=0)$ in the operated leg, while in the intramedullary fixation group, the mean VAS was 1.2 (range $0-8)$ and eight patients (38\%) had no pain in the operated leg.

The IMN group achieved better Lequesne Algofunctional scores $(p=0.006)$. The mean Lequesne Algofunctional score was $>14$ (ranging from 9.5 to 23 ) in the EF group and 11.2 (ranging from 3.5 to 20.5) in the IM group (Table 1).

Regarding to patient's satisfaction, in the Ex Fix group, we found that five patients $(50 \%)$ were very satisfied with the final outcome: four patients $(40 \%)$ were fairly satisfied 


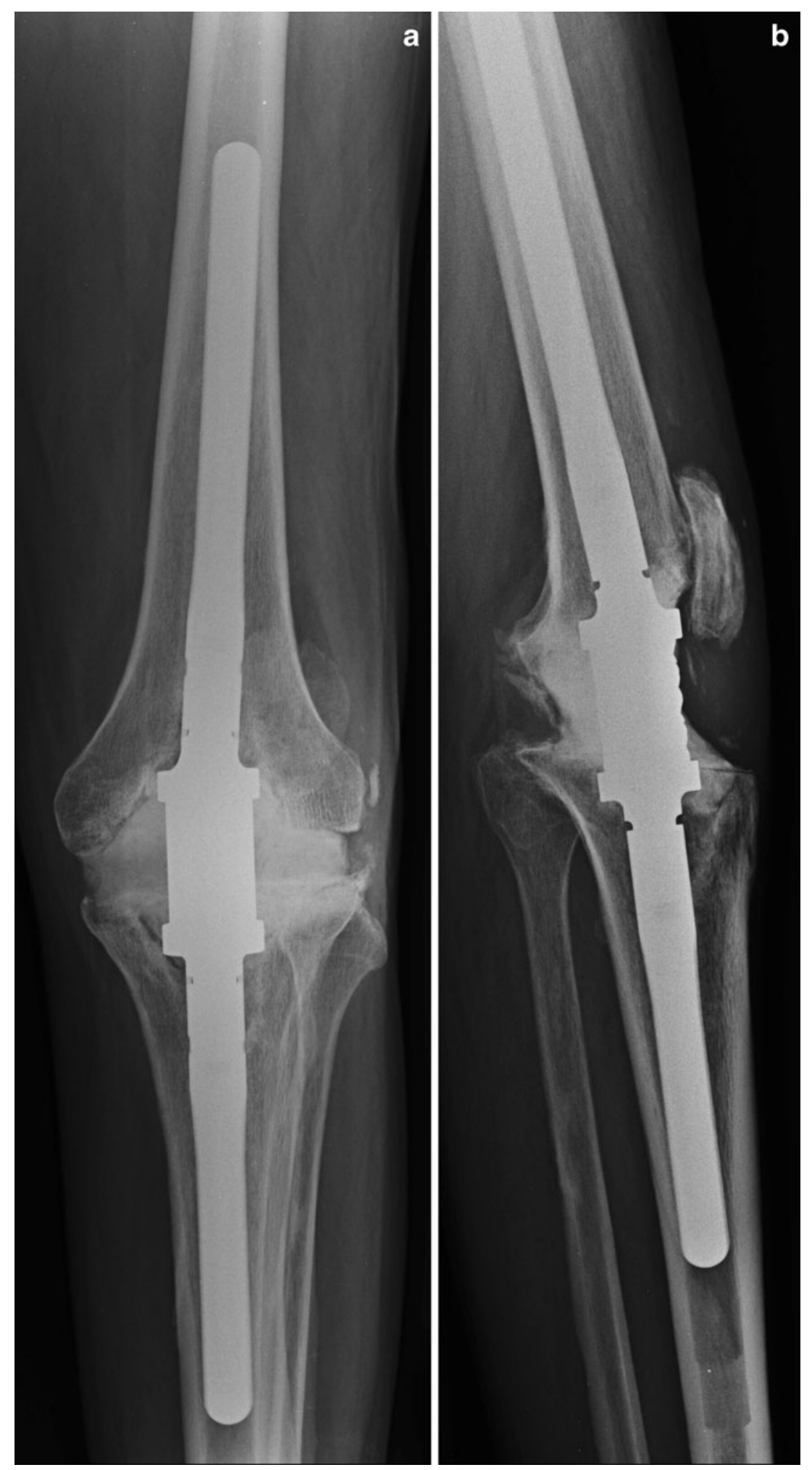

Fig. 2. Postoperative radiographs in frontal (a) and lateral (b) view at 3 months after intramedullary arthrodesis showing maintenance of joint space filled with antibiotic-impregnated cement spacer to preserve leg length. No attempt to achieve bony union.

and one patient (10\%) was very unsatisfied. In the IMN group, 12 patients $(66.7 \%)$ were very satisfied with the outcome, 5 patients $(27.7 \%)$ were fairly satisfied, and 1 patient $(5.6 \%)$ was very unsatisfied.

\section{Discussion}

The primary treatment goal of arthrodesis for an infected RTKA is eradication of infection and maintenance of patient 
Table 1 Comparison of results between patients treated with intramedullary (IM) nail and external fixator (EF)

\begin{tabular}{|c|c|c|}
\hline & IM nail & $\mathrm{EF}$ \\
\hline Number of patients & $22(1$ dead $)$ & $12(2$ dead $)$ \\
\hline Mean FU (range) & 34.4 (13-72 months) & 93.2 (82-110 months) \\
\hline Age (range) & $69.3(53-85)$ & $68.5(55-84)$ \\
\hline Mean numbers of previous surgery & $4.6(1-6)$ & $3.87(2-6)$ \\
\hline No. of recurrent infection & $3(14.28 \%)$ & 0 \\
\hline Mean VAS (range) & $1.2(0-8)$ & $1.9(0-7)$ \\
\hline Mean Lequesne Algofunctional Index (range) & $11.2(3.5-20.5)$ & $>14(9.5-23)$ \\
\hline Pain in ipsilateral or controlateral joint & $\begin{array}{l}-2 \text { Ipsilateral hip } \\
-2 \text { Controlateral knee } \\
-6 \text { Back pain }(33 \%)\end{array}$ & $\begin{array}{l}-2 \text { Ipsilateral hip } \\
-2 \text { Controlateral knee } \\
-1 \text { Ipsilateral hip and controlateral knee } \\
-6 \text { Back pain }(60 \%)\end{array}$ \\
\hline Use of walking devices & $\begin{array}{l}\text {-No } 5 \text { patient }(27.7 \%) \\
\text {-Use of cane } 11 \text { patients }(61.1 \%) \\
\text {-Use of crutches } 2 \text { patients }(11.2 \%)\end{array}$ & $\begin{array}{l}\text {-No } 1 \text { patient }(10 \%) \\
\text {-Use of cane } 7 \text { patients }(70 \%) \\
\text {-Use of crutches } 2 \text { patients }(20 \%)\end{array}$ \\
\hline Mean limb shortening (range) & $0.8(0-22 \mathrm{~cm})$ & $4.5(3.5-5.5 \mathrm{~cm})$ \\
\hline Patients satisfaction & $\begin{array}{l}-12 \text { patients }(66.7 \%) \text {, very satisfied } \\
-5 \text { patients }(27.7 \%) \text {, fairly satisfied } \\
-1 \text { patient }(5.6 \%), \text { unsatisfied }\end{array}$ & $\begin{array}{l}-5 \text { patients }(50 \%) \text {, very satisfied } \\
-4 \text { patients }(40 \%) \text {, fairly satisfied } \\
-1 \text { patient }(10 \%) \text {, unsatisfied }\end{array}$ \\
\hline Mean time to fusion & Not evaluated & 5.6 (3.5-11 months) \\
\hline
\end{tabular}

function. The treatment of an infected TKA remains a challenging problem: soft tissue damage, severe bone loss, and extensor mechanism deficiency secondary to recurrence of infection after failed revision TKA may compromise the knee and a reimplantation has a high chance of failure [8, 10, 19, 20, 23, 28]. Knee arthrodesis performed with an external fixator or IM nail represents a valid solution in patients treated for failed TKA secondary to infection and are not able to further revision arthroplasty. Both these techniques fuse the joint through direct bone to bone apposition failing to preserve limb length.

This study reports our results achieved in a cohort of 34 patients dealt with both techniques. IM nail was used as an endoprosthesis to avoid shortening of operated leg. In fact in this case nonunion and no bone contact is required so the surgeon is able to recreate the desired limb length.

The aim of this retrospective study was to compare these two techniques following infected R-TKA. Recurrence of infection, leg length discrepancy, pain, function results, and patient's satisfaction were evaluated. A successful infection eradication was observed in 28 of 31 patients treated with arthrodesis. No recurrence of infection was observed in the EF group while there were three recurrent infections in the IM nail group. The mean leg length discrepancy was significantly greater in the first group than in the second one. Clinical and functional results showed a substantial improvement relative to preoperative condition in both groups. However, function (LAS) was significantly better in the IM nail group. More than $90 \%$ of patients of both groups were satisfied with the operation.

There are several important limitations of this study. First, the indication to treat the patient either an EF or an IM nail was based on the technique we used at the time of surgery and ideally should have been randomized. Second, though the two groups were homogeneous regarding indication for arthrodesis, they included a different number of patients followed for different periods of time, reducing the statistical power and failing to reach any statistically significant difference in outcome. Third, no interobserver reliability for results at final evaluation was performed. Finally, the follow-up for patients treated with IM nail was short so the risk of long-term implant failure was not studied.

The patients described in this study represent a homogeneous population for indication to arthrodesis. In all cases, in fact, diagnosis was infection after a R-TKA for previous infected TKA. Infection was eradicated in $90 \%$ of the patients in this study. These data are in agreement with the literature where recurrence rates range from 0 to $20 \%[2,5$, $18,27]$. The rate of infection recurrence was significantly higher in the IM nail group (14\%) compared to EF group $(0 \%)$. An earlier study by Mabry et al. [16] reported a higher rate of recurrent infection with IM nailing when compared to external fixation. Although some authors [2, 11, 12, 27] recommend use of the two-stage technique when using an IM nail, other authors have demonstrated that a one-stage procedure with the use of intramedullary nail was safe and with no recurrent infection [3, 21].

In our study, we used a two-stage arthrodesis in all 34 cases. In fact, the presence of active infection may increase both the risk of nonunion when $\mathrm{EF}$ is used $[13,30]$ and recurrent and catastrophic infection because of its potential spreading into femur and tibia (pandiaphysitis) when IM nail is used [16, 24].

Arthrodesis was performed when the knee was clinically free of any conventional sign of infection and when ESR and CRP were reduced relative to values before first stage. When an infection was suspected intraoperatively, the knee joint was further debrided and the cement spacer exchanged. After arthrodesis, intravenous antibiotics according to the culture's sensitivity test or empirically for 4 weeks followed by oral antibiotics for 2 weeks were routinely used. Although there is no consensus on the use of antibiotics postoperatively, we think that their use as well as surgical debridement and antibiotic-cemented spacer may have been helpful to reduce the rate of infection recurrence. 


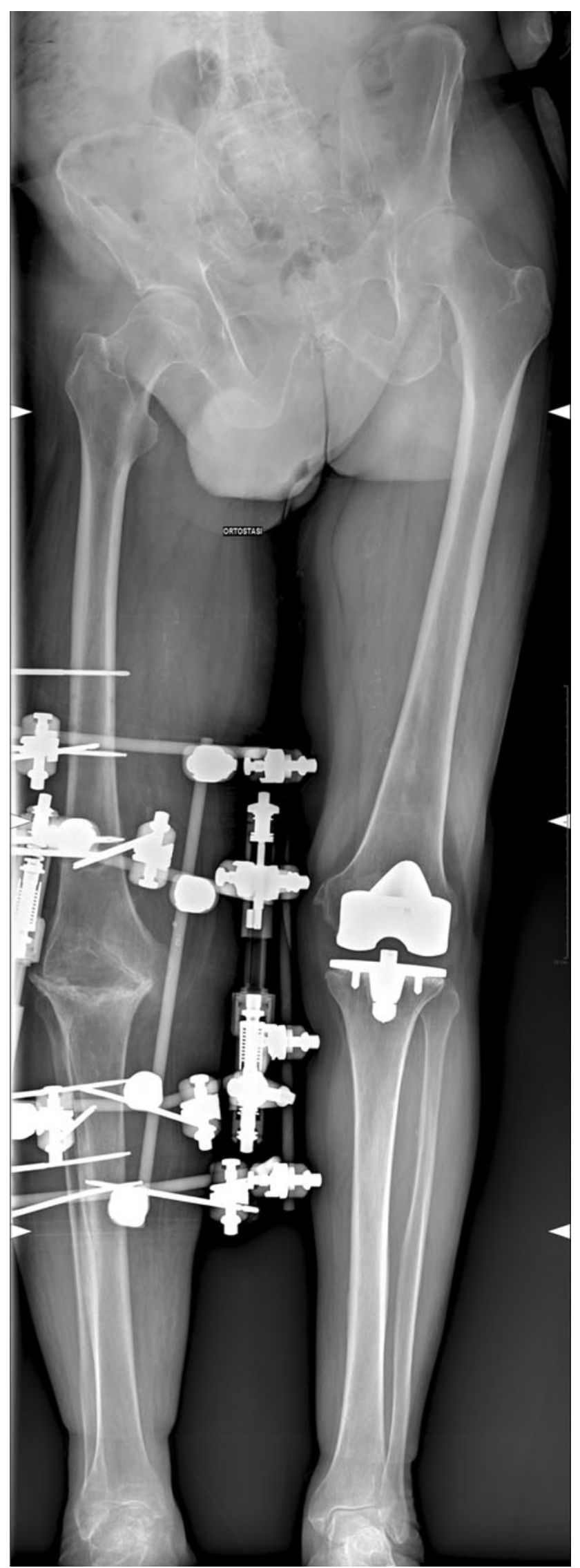

Fig. 3. Long leg standing X-ray showing a shortening of $5 \mathrm{~cm}$ after arthrodesis with EF.
The technique used in the IM nail group allowed us to avoid shortening more than $3 \mathrm{~cm}$, which has been associated with a significant increase in physiologic demand when walking [7]. In fact, as no union, and therefore no bone contact, is required when using an IM nail like prosthesis, the limb length was preserved. Our results are comparable to results of other authors who used the nail as a prosthesis [15, 22].

Although fusion rates after external fixation are lower than those following IM fixation in literature [6, 18, 26, 27, 29], we could not compare our cohorts for fusion rate.

Fusion in nine out of ten patients treated with EF (90\%) was achieved and a painless nonunion in one case. This result is better than the fusion rate of $64 \%$, reported in the literature with a biplanar fixation [4]. We think it is due to the optimal bone contact between the femoral and tibial segments, the accurate debridement of joint surfaces, and the eradication of infection prior to arthrodesis, as well as compression obtained using the EF. We preferred the Hoffmann II Stryker external device because of its easy application, excellent stability, and versatility in terms of limb alignment. In addition, compression was progressively adjusted by compression rods according to radiographs performed after operation.

In the present study, data on VAS and LAS were improved respect to preoperative condition without statistical significant difference between the two groups. Patients with IM nail arthrodesis seem to function better than EF. In fact, they had a significant lower Lequesne Score or a lower severity of handicap in their daily life and were able to walk with no aid in $27.7 \%$ of cases compared to $10 \%$ of EF group. We think that these results are correlated to marked limb length discrepancy observed in patients treated with EF (Fig. 3).

Patient satisfaction was good and comparable in the two groups, with more than $90 \%$ of patients satisfied with the operation.

Although the surgeon and the patient may consider an arthrodesis to be a poor outcome, a successful arthrodesis with a permanently immobilized and stable extremity is associated with a remarkable relief of pain, a reasonably good functionality comparable with that of a revised hinged TKA [5]. In the present study, a successful eradication of infection with satisfactory clinical and functional results was observed in both groups of patients. Although recurrence of infection in our study was lower in patients treated with $\mathrm{EF}$, the two-stage arthrodesis with IM nail, without bone fusion, produced a stable, painless, and functional limb with preserved limb length. The risk of recurrent infection is higher with this technique and there is a possibility of diffused femoro-tibial contamination. Therefore, authors reserve the $\mathrm{EF}$ to cases of residual and persistent infection with positive cultures after prosthesis removal.

\section{Disclosures}

Conflict of Interest: Iacono Francesco, MD, Raspugli Giovanni Francesco, MD, Bruni Danilo, MD, Lo Presti Mirco, MD, Sharma Bharat, MD, Akkawi Ibrahim, MD, and Marcacci Maurilio, MD have declared that they have no conflict of interest. 
Human/Animal Rights: All procedures followed were in accordance with the ethical standards of the responsible committee on human experimentation (institutional and national) and with the Helsinki Declaration of 1975, as revised in 2008 (5).

Informed Consent: Informed consent was waived from all patients for being included in the study.

Required Author Forms Disclosure forms provided by the authors are available with the online version of this article.

\section{References}

1. Azzam K, McHale K, Austin M, Purtill JJ, Parvizi J. Outcome of a second two-stage reimplantation for periprosthetic knee infection. Clin Orthop Relat Res. 2009; 467: 1706-1714.

2. Bargiotas K, Wohlrab D, Sewecke JJ, Lavinge G, Demeo PJ, Sotereanos NG. Arthrodesis of the knee with a long intramedullary nail following the failure of a total knee arthroplasty as the result of infection. J Bone Joint Surg Am. 2006; 88: 553-558.

3. Conway JD, Mont MA, Bezwada HP. Arthrodesis of the knee. $J$ Bone Joint Surg Am. 2004; 86: 835-848.

4. Damron TA, Mc Beath AA. Arthrodesis following failed total knee arthroplasty: comprehensive review and meta-analysis of recent literature. Orthopedics. 1995; 18: 361-368.

5. De Vil J, Almquist KF, Vanheeren P, Boone B, Verdonk R. Knee arthrodesis with an intramedullary nail: a retrospective study. Knee Surg Sport Traumatol Arthrosc. 2008; 16: 545-550.

6. Ellingsen DE, Rand JA. Intramedullary arthrodesis of the knee after failed total knee arthroplasty. J Bone Joint Surg Am. 1994; 7676: 870-876.

7. Gurney B, Mermier C, Robergs R, Gibson A, Rivero D. Effects of limb-length discrepancy on gait economy and lower extremity muscle activity in older adults. J Bone Joint Surg Am. 2003; 83A: 907-915.

8. Hanssen AD, Trousdale RT, Osmon DR. Patient outcome with reinfection following reimplantation for the infected total knee arthroplasty. Clin Orthop. 1995; 321: 55-67.

9. Harris IE, Leff AR, Gitelis S, Simon MA. Function after amputation, arthrodesis, or arthroplasty for tumors about the knee. J Bone Joint Surg Am. 1990; 72: 1477-1485.

10. Hirakawa K, Stulberg BN, Wilde AH, Bauer TW, Secic M. Results of 2-stage reimplantation for infected total knee arthroplasty. $J$ Arthroplasty. 1998; 13(1): 22-28.

11. Iacono F, Bruni D, Lo Presti M, et al. Knee arthrodesis with a press-fit modular intramedullary nail without bone-on-bone fusion after an infected revision TKA. The Knee. 2012; 19(5): 555-559.

12. Knutson K, Hovelius L, Lindstrand A, Lidgren L. Arthrodesis after failed knee arthroplasty. A nationwide multicenter investigation of 91 cases. Clin Orthop. 1984; 191: 202-211.
13. Lai KA, Shen WJ, Yang CY. Arthrodesis with a short Huckstep nail as salvage procedure for failed total knee arthroplasty. $J$ Bone Joint Surg. 1998; 80-A: 380-388.

14. Lequesne M, Mery C, Samson M, Gerard P. Indexes of severity for osteoarthritis of the hip and knee. J Rheumatol. 1995; 65: 85-89.

15. Letartre R, Combes A, Autissier G, Bonnevialle N, Gougeon F. Knee arthodesis using a modular customized intramedullary nail. Orthop Traumatol Surg Res. 2009; 95(7): 520-528.

16. Mabry TM, Jakofsky DJ, Haidukewych GJ, Hanssen AD. Comparison of intramedullary nailing and external fixation knee arthrodesis for the infected knee replacement. Clin Orthop. 2007; 464: 11-15.

17. Maheshwari AV, Gioe TJ, Kalore NV, Cheng EY. Reinfection after prior staged reimplantation for septic total knee arthroplasty: is salvage still possible? J Arthroplasty. 2010; 25(6 Suppl): 92-7.

18. McQueen DA, Cooke FW, Hahn DL. Knee arthrodesis with the Wichita Fusion Nail: an outcome comparison. Clin Orthop. 2006; 446: 132-139.

19. Mittal Y, Fehring TK, Hanssen A, Marculescu C, Odum SM, Osmon D. Two-stage reimplantation for periprosthetic knee infection involving resistant organisms. J Bone Joint Surg Am. 2007; 89(6): 1227-1231.

20. Mont MA, Waldman BJ, Hungerford DS. Evaluation of preoperative cultures before second-stage reimplantation of a total knee prosthesis complicated by infection. A comparison-group study. $J$ Bone Joint Surg Am. 2000; 82-A(11): 1552-1557.

21. Puranen J, Kortelainen P, Jalovaara P. Arthrodesis of the knee with intramedullary nail fixation. J Bone Joint Surg Am. 1990; 72(3): 433.

22. Rao MC, Richards O, Meyer C, Spencer JR. Knee stabilisation following infected knee arthroplasty with bone loss and extensor mechanism impairment using a modular cemented nail. The Knee. 2009; 16: 489-493.

23. Salgado CD, Dash S, Cantey JR, Marculescu. Higher risk of failure of methicillin-resistant Staphylococcus aureus prosthetic joint infections CE. Clin Orthop Relat Res. 2007; 461: 48-53.

24. Talmo CT, Bono JV, Figgie MP, Sculco TP, Laskin RS, Windsor RE. Intramedullary arthrodesis of the knee in the treatment of sepsis after TKR. HSSJ. 2007; 3: 83-88.

25. Utting MR, Newman JH. Customised hinged knee replacements as a salvage procedure for failed total knee arthroplasty. Knee. 2004; 11(6): 475-479.

26. Volpi R, Dehoux E, Touchard P, Mensa C, Segal P. Arthrodese du genou par implant intra medullaire sur mesure. A propos de 14 cas. Rev Chir Orthop. 2004; 90: 58-64.

27. Waldman BJ, Mont MA, Payman KR, et al. Infected total knee arthroplasty treated with arthrodesis using a modular nail. Clin Orthop. 1999; 367: 230-237.

28. Wasielewski RC, Barden RM, Rosemberg AG. Results of different surgical procedures on total knee arthroplasty infections. $J$ Arthroplasty. 1996; 11: 931-938.

29. Wiedel JD. Salvage of infected total knee fusion: the last option. Clin Orthop. 2002; 404: 139-142.

30. Wilde AH, Stearns KL. Intramedullary fixation for arthrodesis of the knee after infected total knee arthroplasty. Clin Orthop Relat Res. 1989; 248: 87-92. 